Slow topological time scale of knotted polymers

Non Peer-reviewed author version

Orlandini, Enzo; Stella, Attilio L.; VANDERZANDE, Carlo \& Zonta, Francesco (2008)

Slow topological time scale of knotted polymers. In: JOURNAL OF PHYSICS

A-MATHEMATICAL AND THEORETICAL, 41(12).

DOI: $10.1088 / 1751-8113 / 41 / 12 / 122002$

Handle: http://hdl.handle.net/1942/8191 


\title{
Slow topological time scale of knotted polymers
}

\author{
Enzo Orlandini ${ }^{1,2}$, Attilio L. Stella ${ }^{1,2}$, Carlo Vanderzande ${ }^{3,4}$ and \\ Francesco Zonta ${ }^{5}$ \\ ${ }^{1}$ Dipartimento di Fisica and CNR-INFM, Università di Padova, I-35131, Padova, \\ Italy \\ ${ }^{2}$ Sezione INFN, Università di Padova, I-35131 Padova, Italy \\ ${ }^{3}$ Departement WNI, Hasselt University, 3590 Diepenbeek, Belgium \\ ${ }^{4}$ Instituut Theoretische Fysica, Katholieke Universiteit Leuven, 3001 Heverlee, \\ Belgium \\ ${ }^{5}$ Dipartimento di Fisica, Università di Padova, I-35131, Padova, Italy \\ E-mail: carlo.vanderzande@uhasselt.be
}

\begin{abstract}
We investigate the Rouse dynamics of a flexible ring polymer with a prime knot. Within a Monte Carlo approach, we locate the knot, follow its diffusion, and observe the fluctuations of its length. A topological time scale that is slower then the Rouse one, is found to determine the long time behaviour of several dynamical quantities. The value of the associated dynamical exponent is $z_{T}=2.32 \pm .1$.
\end{abstract}

PACS numbers: 36.20.Ey, 64.60.Ht, 02.10.Kn, 87.15.Aa 
In the physics of polymers, mutual and self entanglements play an essential role [1]. A particularly relevant type of entanglement is associated with the presence of knots. Indeed, it has been known for some time that long ring polymers inevitably contain a knot [2]. Topology is also of much interest for biopolymers, where knots have been found in the DNA of viruses and bacteria [3], and also in some proteins [4].

The recent experimental possibility to tie knots in DNA double strands or actin filaments [5], and to study their diffusion in the presence of a stretching force [6], has further increased the interest in topology related issues among polymer physicists $[7,8,9]$.

So far, the statistical physics of knotted polymers has focused mostly on static properties [10]. For example, it was found that the presence of a knot does not alter the exponent $\nu$ that relates the radius of gyration $R_{G}(N)$ to the length $N$ of the polymer, $R_{G}(N) \sim N^{\nu}$, but only influences scaling corrections $[11,12]$. There have been fewer studies of dynamical scaling properties of knotted polymers, even though the associated time scales could be relevant to describe gel electrophoresis, folding of knotted proteins or other dynamical processes. Simulations have given evidence that a peculiar, topological, characteristic time $\tau_{T}$ determines the decay of the radius of gyration autocorrelation function of such polymers $[13,14]$. This decay time appears longer than that observed for open or closed unknotted chains. As a function of chain length, it was found to scale with a dynamical exponent $z_{T}$, that could not be determined precisely but whose value probably is bigger than the Rouse one. So far, the physical origin of this time was not understood.

In this Letter, we investigate how a knot influences dynamical scaling properties of flexible polymers in a good solvent within the framework of Rouse dynamics $[1,15]$. In a simulation, we directly follow the diffusion of the knotted region and determine the fluctuations of its length (measured along the backbone). The present work is the first one to apply a recently introduced technique to locate a knot in a polymer [12] in a study of dynamical properties. On the basis of a diffusion picture, we conjecture a link between $z_{T}$ and the exponent governing the equilibrium distribution of this length.

In Rouse dynamics, one models a polymer as a set of $N+1$ beads (monomers) that are connected by harmonic springs, and that are subjected to random thermal forces exerted by the solvent. The motion of the monomers is described by a Langevin equation, which for ideal chains can be solved using a transformation to normal coordinates. If self-avoidance is taken into account, Rouse dynamics can no longer be solved exactly, but scaling arguments together with numerical results [16], show that the center of mass of the polymer, $\boldsymbol{R}_{c m}$, performs ordinary diffusion, i.e.

$$
g_{3}(t, N) \equiv\left\langle\left(\boldsymbol{R}_{c m}(t)-\boldsymbol{R}_{c m}(0)\right)^{2}\right\rangle \simeq 6 D_{c m} t
$$

where the average is taken over realisations of the stochastic process. The diffusion constant is inversely proportional to the length $N$ of the polymer, i.e. $D_{c m} \sim N^{-1}$. The autocorrelation function of the end-to-end vector decays exponentially with a time 
constant $\tau_{R}$ that grows as

$$
\tau_{R} \sim N^{2 \nu+1}
$$

with $N$. In $d=3,2 \nu+1 \simeq 2.2$ [16]. We will refer to $\tau_{R}$ as the Rouse time scale. It can be interpreted as the time that the center of mass of the polymer needs to diffuse over a length equal to its radius of gyration, $\tau_{R} \sim R_{G}^{2}(N) / D_{c m} \sim N^{2 \nu+1}$.

The diffusion of one particular monomer, or of a segment of $m+1$ monomers, is described by the function

$$
g_{1}(t, N)=\frac{1}{m+1} \sum_{i=(N-m) / 2}^{(N+m) / 2}\left\langle\left(\boldsymbol{R}_{i}(t)-\boldsymbol{R}_{i}(0)\right)^{2}\right\rangle .
$$

It is known [17] that $g_{1}(t)$ has a scaling form

$$
g_{1}(t, N) \simeq t^{\beta} F\left(t / \tau_{R}\right) .
$$

The function $F$ describes the crossover between an initial power law regime $(F(x) \rightarrow$ constant, for $x \rightarrow 0$ ) and a late time regime for which a monomer has to follow the diffusion of the whole polymer as given by (1). This implies a power law form for $F$ at late times and $\beta=2 \nu /(2 \nu+1)$, i.e. in the initial regime the movement of a segment of the polymer is subdiffusive. Notice that $g_{1}\left(\tau_{R}, N\right) \sim R_{G}^{2}(N)$, so that the Rouse time scale also corresponds to the time for one monomer to diffuse over a distance equal to $R_{G}(N)$. In the whole dynamics, it is the only relevant time.

Rouse dynamics neglects important physical effects such as hydrodynamic interactions between the monomers. These are taken into account in the Zimm model [1] for which it is found that the slowest relaxation time becomes proportional to $N^{3 \nu}$. In the present paper, we will concentrate on Rouse dynamics. Several experimental situations are known by now for which it gives an adequate description. As an example, we mention that current fluorescence techniques allow to follow the motion of individual monomers in, e.g., DNA-chains and hence to directly determine a function such as $g_{1}(t, N)$. Measurements of this type on double stranded DNA have recently seen diffusive behaviour of individual monomers consistent with Rouse behaviour [18]. DNA adsorbed on a lipid membrane is another system of current interest that can be understood in terms of Rouse dynamics [19].

A crucial quantity to characterise the dynamics of a knotted polymer turns out to be the length of the knot. A precise definition for this quantity can be given for flat knots [20]. These are knots in a polymer that is strongly adsorbed to a plane or constrained between two walls. In this context, it was found that the length $l_{k}$ of a knot $k$ is a fluctuating quantity, whose equilibrium distribution function is a power law, $p\left(l_{k}\right) \sim l_{k}^{-c} G\left(l_{k} / N\right)$ where $G$ is a scaling function. It follows, that the average length of the knot scales with $N$ as $\left\langle l_{k}\right\rangle \sim N^{\sigma}$, with $\sigma=\max [0,2-c]$. In a good solvent, flat knots were found to be strongly localised $(\sigma=0)$ [20], while below the $\theta$-transition, they delocalise $(\sigma=1)$ [21]. In order to extend these results to three dimensions, one needs a good definition of the length of a knot. This should correspond to the intuitive idea that it measures the portion of the polymer backbone which "hosts" the knot entanglement 
[22]. Recently, a powerful computational approach to determine such a length, and its scaling properties, was introduced [12]. In this method, for a given knotted ring polymer, various open portions are considered and for each of these a closure is made by joining its ends with an off-lattice path. The length of the knot in a given configuration can then be identified with the shortest portion still displaying the original knot. In this way, it was found that in good solvent, three dimensional knots are weakly localised with an exponent $\sigma=.75 \pm .05$.

To simulate the dynamics of knotted polymers, we start from a simple self-avoiding polygon (SAP) on a cubic lattice with a knot in it. Most of our calculations were done with a trefoil $\left(3_{1}\right)$ knot. We used the BFACF algorithm [23] to relax the configuration. This algorithm works in the grand canonical ensemble, and is known to preserve topology. The resulting SAP is then evolved according to a $N$-conserving MonteCarlo dynamics with local moves only. During the subsequent evolution, using the computational techniques developed in [12], observables such as the length of the knot, the radius of gyration of the whole polymer, the location of the center of mass of the polygon and the location of the center of mass of the knot, $\boldsymbol{R}_{c m, k}$, are computed. We have performed calculations for various $N \leq 400$.

It can be questioned whether the restriction to a lattice provides a correct description of continuum dynamics. This problem has, amongst others, been studied in [24] where it was found that in the presence of excluded volume effects, the $N$ dependence of the relaxation times of the first three Rouse modes is consistent with the prediction from scaling theory. In order to verify our results we have also performed a simulation of the dynamics of a knotted polymer (of 100 monomers) using a Langevin method [25]. The results agree both qualitatively and quantitatively with those coming form the simpler, and faster, lattice algorithm used in the present work.

From the data on the knot length as a function of time, we can construct the knot length autocorrelation function $\left\langle l_{k}(t) l_{k}(0)\right\rangle_{c}$ (where the subscript 'c' indicates the properly normalised connected autocorrelation). In figure 1, the top (red) line shows our results for this quantity for $N=400$. This autocorrelation function has a simple exponential decay. Similar behaviour is found for other $N$ values. Figure 2 presents our data for the decay time constants as a function of $N$. The behaviour is power law, and the exponent is $2.33 \pm 0.08$. The value of this exponent is higher than that of Rouse dynamics. Further evidence that this presents a new time scale comes from an investigation of the autocorrelation function of the radius of gyration of the polymer. In figure 1 we also plotted our results for this quantity (bottom line). Clearly, it has a double exponential decay. In comparison, for an unknot, we observe a pure exponential decay. A careful analysis shows that the time constant of the unknot, together with the fast one of the knotted polymer, are proportional to $\tau_{R}$ (figure 2). On the other hand, as can be seen in figure 1, the late time decay of the radius of gyration autocorrelation occurs with the same time constant as that of the length autocorrelation. A quantitative analysis of its $N$-dependence supports this conclusion (see figure 2). Indeed, the value of the associated exponent equals $2.31 \pm 0.08$, consistent within the numerical accuracy 


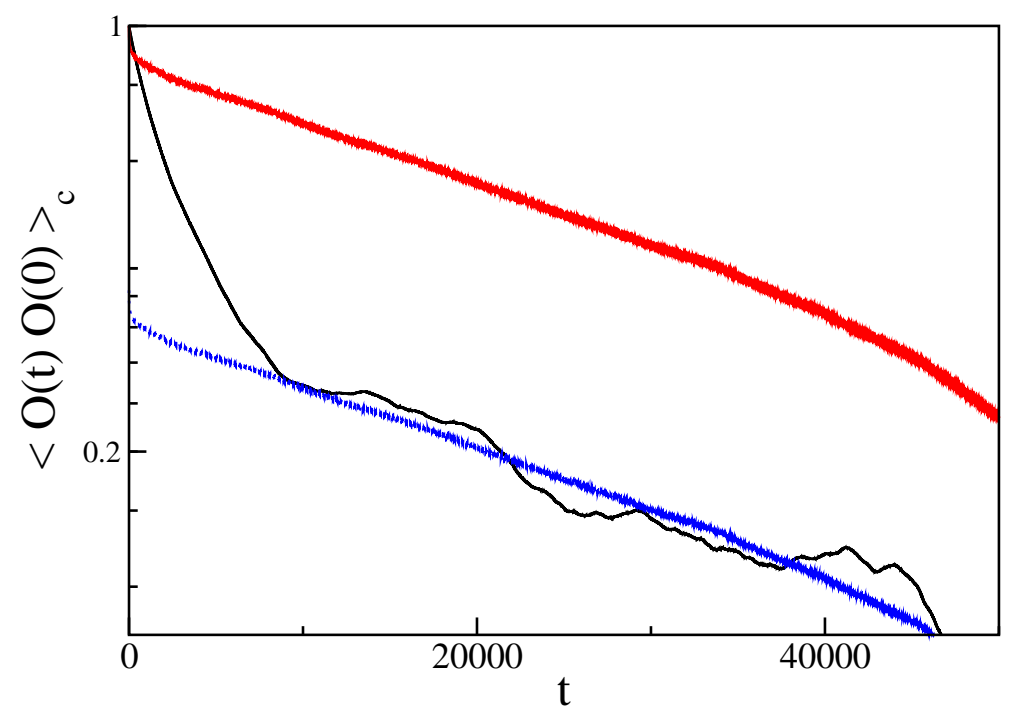

Figure 1. (Color online) Semilogarithmic plot of the autocorrelation functions versus time for a SAP with $N=400$. Shown are results for the radius of gyration (black, below) and the knot length (red, above). The blue line was obtained by shifting the red one vertically.

with that determined from the length autocorrelation. Together, these data therefore provide strong evidence for the presence of a new, slow time scale $\tau_{T} \sim N^{z_{T}}$, with $z_{T}=2.32 \pm .1$, in the dynamics of knotted polymers. The data on the knot length autocorrelation show that this scale corresponds to the time over which the knot length decorrelates.

To check if the results are influenced by the topology of the knot, we have performed a completely similar study for the figure-eight $\left(4_{1}\right)$ knot. Again the radius of gyration autocorrelation function decays as a double exponential, with a fast time scale that can be identified with $\tau_{R}$. The slower time scale grows with $N$ with an exponent whose numerical value, $2.33 \pm .08$ is consistent with that found for the trefoil. Moreover, the same exponent was found to govern the decay of the $4_{1}$ length autocorrelation function.

In order to determine whether the topological time also influences other dynamical properties, we have investigated the diffusion of the center of mass of the whole polymer and of the knotted region. From our data, we determine $g_{3}(t, N)$ and a similar quantity for the knotted part of the polymer

$$
g_{3, k}(t, N)=\left\langle\left(\boldsymbol{R}_{c m, k}(t)-\boldsymbol{R}_{c m, k}(0)\right)^{2}\right\rangle
$$

This function has some similarity to $g_{1}(t, N)$ since it describes the motion of a segment of the polymer. Hence we can expect it to have a scaling behaviour similar to (4). There are however differences since the number of monomers in the knot increases with $N^{\sigma}$ on 


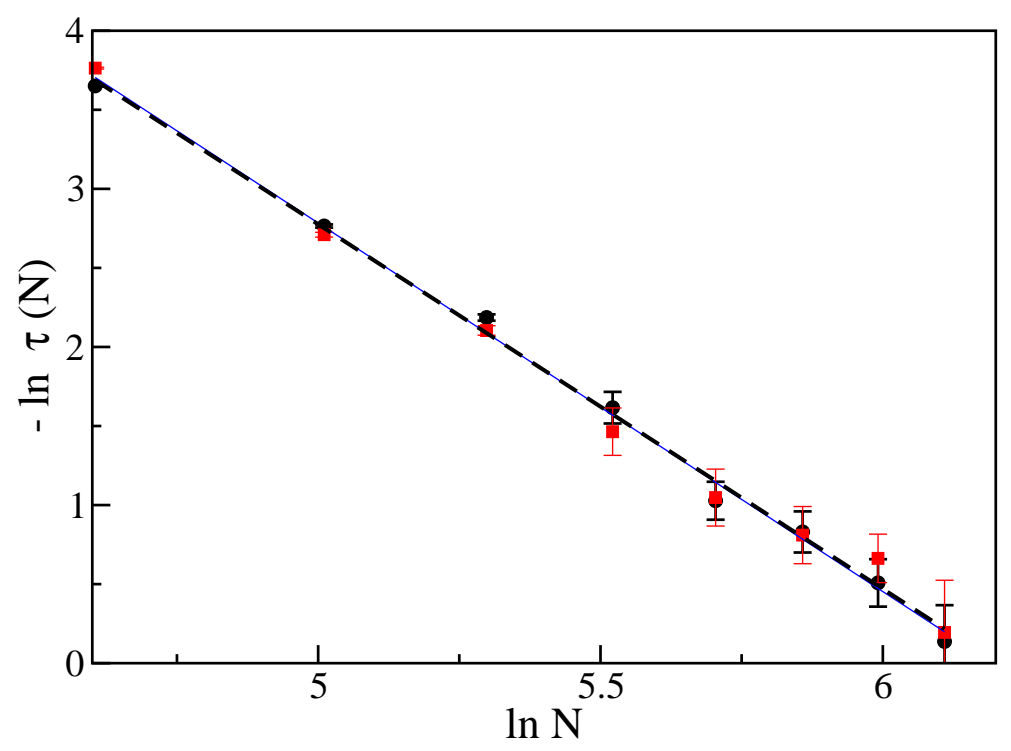

Figure 2. (Color online) Log-log plot of the topological time scale versus $N$ as determined from the radius of gyration autocorrelation function (black circles) and from the knot length autocorrelation (red squares). Also shown are the time constants associated with the early time decay of the radius of gyration autocorrelation (...). The fitted lines have slopes $-2.31 \pm 0.08$ (black dashed line) and $-2.33 \pm 0.08$ (blue full line) respectively.

average, and, at fixed $N$, fluctuates in time.

In figure 3 , we show a typical result $(N=250)$ for the functions $g_{3}(t, N)$ and $g_{3, k}(t, N)$. Our data for the diffusion of the center of mass of the whole polymer are fully consistent with Eq. (1) and with the relation $D_{c m} \sim N^{-1}$, a strong indication that the diffusion of the polymer as a whole is not changed by the presence of the knot [14].

More interesting is the diffusion of the knot itself. As can be seen in figure $3, g_{3, k}$ shows several distinct power law regimes. The value of the estimated exponent for the early time region fluctuates with $N$, but a good average is $0.27 \pm 0.1$. This initial regime ends after a time $\tau_{1}$, which grows as a power of $N, \tau_{1} \sim N^{z_{1}}$, where $z_{1}=1.97 \pm .1$. After a short crossover, in an intermediate time regime, the behaviour is again power law, $g_{3, k} \sim t^{\gamma}$. The value of $\gamma$ decreases from $\approx .81$ for $N=100$ to a value close to .66 at $N=400$. An extrapolation gives the asymptotic value $\gamma=.6 \pm .03$. Finally, and in analogy to the behaviour of $g_{1}$, we expect a crossover of $g_{3, k}$ to linear behaviour, since the knot eventually has to follow the whole polymer. This crossover has not happened yet for the times we were able to simulate. In figure 3 the vertical dashed line indicates the Rouse time, which we have estimated from the relation $g_{3}\left(\tau_{R}, N\right)=R_{G}^{2}(N)$. We checked that the Rouse time calculated in this way indeed grows as $N^{2.2}$. We thus see that, as was the case for the length autocorrelation, $\tau_{R}$ doesn't play a special role for $g_{3, k}$, and moreover, it seems that the second crossover occurs on a much slower time 


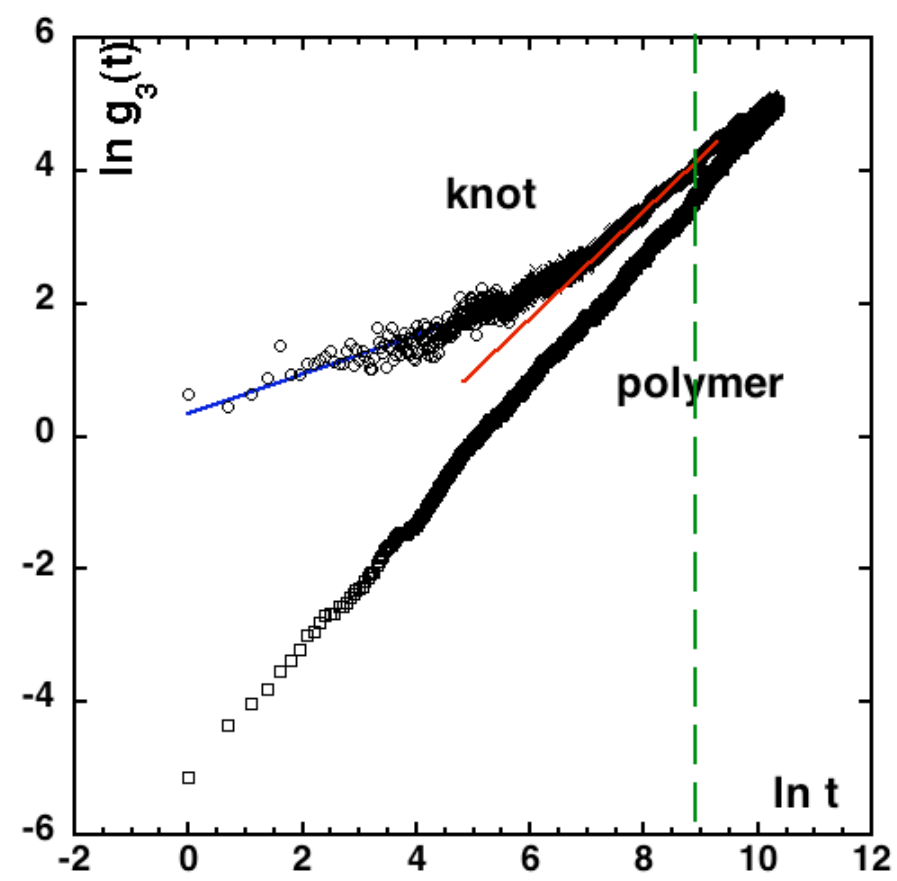

Figure 3. (Color online) The function $g_{3}$ of the whole polymer (lower curve) and of the knot (upper curve) $(N=250)$. The straight lines are best fits through the initial (blue) and intermediate (red) time regime of the knot. Their slopes are .29 and .78, respectively. The dashed vertical line correspond to $\tau_{R}$.

scale, which we expect to be $\tau_{T}$. In analogy with (4), we therefore propose this second crossover to be of the form

$$
g_{3, k}(t, N) \simeq t^{\gamma} H\left(t / \tau_{T}\right) \quad t>\tau_{1}
$$

where $H(x)$ becomes constant for small $x$. Since for $t>\tau_{T}$, the behaviour of (6) must cross over into that of $(1)$, we obtain the relation $(1-\gamma) z_{T}=1$. Using the estimate $z_{T}=2.32 \pm .1$, this leads to $\gamma=.57 \pm .02$ consistent with our estimate. In figure 4, we show a scaling plot of our data for $g_{3, k}(t, N)$ for various $N$-values (leaving out the initial power law regime) and using the values $z_{T}=2.35, \gamma=.57$. The scaling is rather well satisfied. So, the available numerical evidence is consistent with the identification of the second crossover time with $\tau_{T}$.

The Rouse time scale gives the time for the polymer to diffuse over its radius of gyration. We here propose a similar simple interpretation of $\tau_{T}$. Indeed, the presence of the knot introduces a new timescale that is related to its diffusion along the polymer backbone. We assume that this diffusion is normal and that the associated diffusion constant $D_{K}$ is inversely proportional to the knot length, i.e. $D_{K} \sim N^{-\sigma}$ (see [7] for a similar assumption). We then identify $\tau_{T}$ as the time it takes for this diffusion to move over the length of the knotted region. This gives $\tau_{T} \sim N^{2 \sigma} / D_{K} \sim N^{3 \sigma}$. Taking 


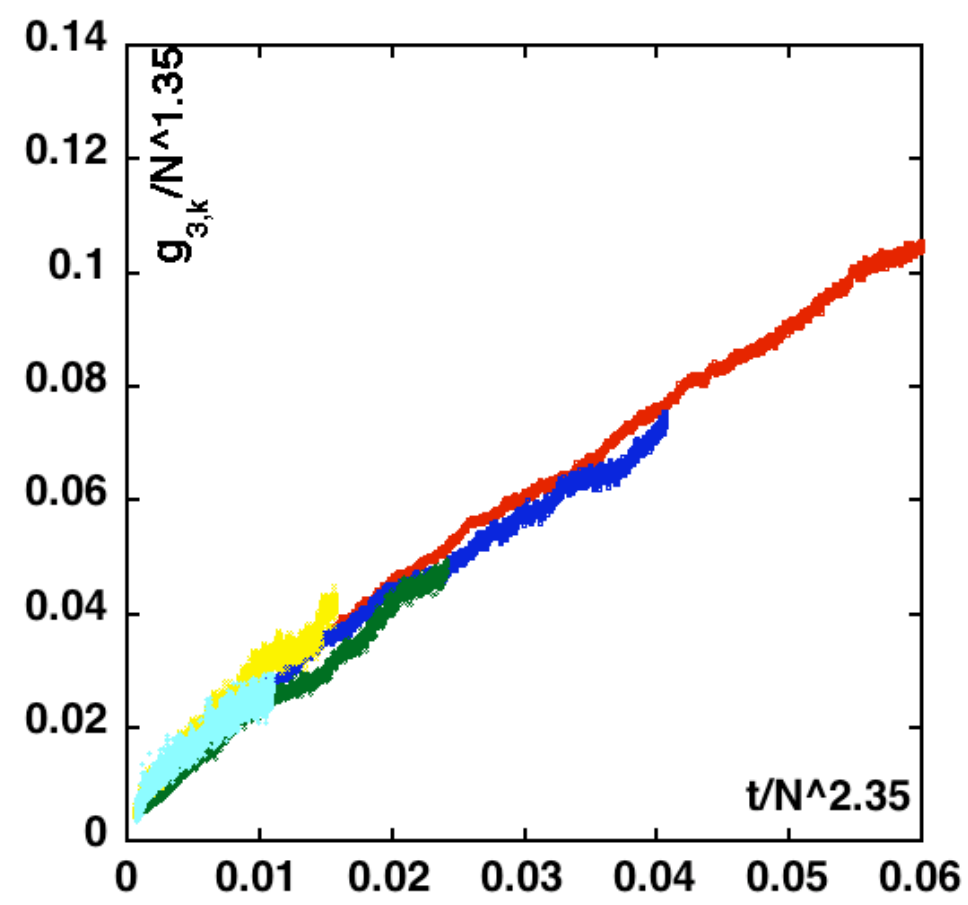

Figure 4. (Color online) Scaling plot of our results for $g_{3, k} / N^{z_{T} \gamma}$ versus $t / N^{z_{T}}$ for various $N$-values using $z_{T}=2.35, \gamma=.57$. Different colors correspond to different $N$ values, red: $N=150$, blue: 200, dark green: 250 , yellow: 300 and light green: 350 .

the estimate of $\sigma$ from [12], we find $3 \sigma=2.25 \pm .15$, which is consistent with the identification $z_{T}=3 \sigma$. This agreement supports the plausibility of our argument.

The same result would be obtained by assuming that the knotted region moves by reptation. Indeed, reptation of a polymer in a melt introduces a slow time scale $\tau_{d} \sim N^{3}[26]$. Physically $\tau_{d}$ corresponds to the time needed to change the shape of the tube that confines the movement of the polymer. Since the knot length distribution is a fastly decaying power law distribution, the knot will be rather tight for a considerable fraction of time. One can imagine that for these tight conformations, the shape of the knotted region decorrelates by a self-reptation mechanism on a time scale $\left\langle l_{k}\right\rangle^{3} \sim N^{3 \sigma}$. When the knot is looser, its length decorrelates faster. The long time dynamics is then determined by the slowest, self-reptation, mode. We conjecture that the combination of slow, reptative and faster, Rouse-like movement gives rise to a net diffusion with $D_{K} \sim N^{-\sigma}$.

In conclusion, by the first calculation in which dynamical information on the knot itself was monitored, we provided convincing evidence, that the presence of a knot in a ring polymer introduces a new slow topological time scale. This scale can be understood in terms of a diffusion of the knotted region along the polymer backbone. 
Slow topological time scale of knotted polymers

\section{Acknowledgments}

This work was supported by FIRB01, MIUR-PRIN05 and the FWO-Vlaanderen.

\section{References}

[1] Rubinstein M and Colby R H 2003 Polymer Physics (Oxford: Oxford University Press)

[2] Delbruck M 1962 Proc. Symp. Appl. Math 14 55; Sumners D and Whittington S G 1988 J. Phys. A: Math. Gen. 21 1689; Pippenger N 1989 Disc. Appl. Math. 25273

[3] Bates A D and Maxwell A 2005 DNA Topology (Oxford: Oxford University Press)

[4] Taylor W R 2000 Nature 406916 (2000); Virnau P, Mirny L A and Kardar M 2006 PloS Comp. Biol. 21074 (2006); Lua R C and Grosberg A Y 2006 PloS Comp. Biol. 2350

[5] Arai Y, Yasuda R, Akashi K, Harada Y, Miyata H, Kinosita K Jr and Itoh H 1999 Nature 399 446

[6] Bao X R, Lee H J and Quake S R 2003 Phys. Rev. Lett. 91265506

[7] Metzler R, Reisner W, Riehn R, Austin R, Tegenfeldt J and Sokolov I M 2006 Europhys. Lett. 76 696

[8] Huang L and Makarov D E 2007 J Phys Chem A 11110338

[9] Vologodskii A 2006 Biophys. J. 901594

[10] Orlandini E and Whittington S G 2007 Rev. Mod. Phys. 79611

[11] Orlandini E, Tesi M C, Janse van Rensburg E J and Whittington S G 1998 J. Phys. A: Math. Gen. 315953

[12] Marcone B, Orlandini E, Stella A L and Zonta F 2005 J. Phys. A: Math. Gen 38 L15; Marcone B, Orlandini E, Stella A L and Zonta F 2007 Phys. Rev. E 75041105

[13] Quake S R 1995 Phys. Rev. Lett. 733317

[14] Lai P Y 2002 Phys. Rev. E 66021805

[15] Rouse P E 1953 J. Chem. Phys. 211272

[16] P.-G. de Gennes 1979 Scaling concepts in polymer physics (Ithaca: Cornell University Press)

[17] Kremer K and Binder K 1984 J. Chem. Phys. 81 6381; Grest G S and Kremer K, 1986 Phys. Rev. A 333628

[18] Shusterman R, Alon R S, Gavrinyov T and Krichevsky O 2004 Phys. Rev. Lett. 92048303

[19] Maier B and Rädler J O 1999 Phys. Rev. Lett. 821911

[20] Metzler R, Hanke A, Dommersnes P G, Kantor Y and Kardar M 2002 Phys. Rev. Lett. 88188101

[21] Orlandini E, Stella A L and Vanderzande C 2003 Phys. Rev. E 68 031804; A. Hanke, Metzler R, Dommersnes P G, Kantor Y and Kardar M 2003 Eur. Phys. J. E 12347

[22] Katrich V, Olson W K, Vologodskii A, Dubochet J and Stasiak A 2000 Phys. Rev. E 615545

[23] Caracciolo S, Pellissetto A and Sokal A D 1990 J. Stat. Phys. 60 1; Janse van Rensburg E J and Whittington S G 1991 J. Phys. A. 245553

[24] Downey J P, Crabb C C and Kovac J 1986, Macromolecules 192202

[25] Details will be published elsewhere

[26] de Gennes P G 1981 J. Phys. (Paris) 42735 